\title{
Higher dimensional nonclassical eigenvalue asymptotics
}

\author{
Brice Camus. \\ Ludwig Maximilians Universität München, \\ Mathematisches Institut, Theresienstr. 39 D-80803 München. \\ Email: brice.camus@uni-due.de \\ Nils Rautenberg. \\ Ruhr-Universität Bochum, Fakultät für Mathematik, \\ Universitätsstr. 150, D-44780 Bochum, Germany. \\ Email : Nils.Rautenberg@ruhr-uni-bochum.de
}

Revised: July 2, 2018

\begin{abstract}
In this article we extend B. Simon's construction and results 12 for leading order eigenvalue asymptotics to $n$-dimensional Schrödinger operators with non-confining potentials given by: $H_{n}^{\alpha}=-\Delta+\prod_{i=1}^{n}\left|x_{i}\right|^{\alpha_{i}}$ on $\mathbb{R}^{n}$ $(n>2), \alpha:=\left(\alpha_{1}, \cdots, \alpha_{n}\right) \in\left(\mathbb{R}_{+}^{*}\right)^{n}$. We apply the results to also derive the leading order spectral asymptotics in the case of the Dirichlet Laplacian $-\Delta^{D}$ on domains $\Omega_{n}^{\alpha}=\left\{x \in \mathbb{R}^{n}: \prod_{j=1}^{n}\left|x_{j}\right|^{\frac{\alpha_{j}}{\alpha_{n}}}<1\right\}$.
\end{abstract}

Keywords : Trace formulae; Schrödinger operators; Singular asymptotics.

\section{Introduction and main results.}

Since the seminal work of Weyl [14] and its generalizations, the eigenvalue asymptotics of the Laplacian $-\Delta$ on compact domains $\Omega \subset \mathbb{R}^{n}$ with various boundary conditions have been understood to encode information about the geometry of the domain. Let $\left(\lambda_{j}\right)_{j \in \mathbb{N}}$ denote the sequence of eigenvalues of $-\Delta$ on such a domain endowed with Dirichlet boundary conditions. Let furthermore $N(E)=\#\left\{j: \lambda_{j} \leq E\right\}$ be the counting function of eigenvalues. Not even any regularity of the boundary $\partial \Omega$ is required for the Weyl law (see [3]):

$$
N(E)=\frac{\operatorname{vol}\left(S^{n-1}\right)}{(2 \pi)^{n}} \operatorname{vol}(\Omega) E^{n / 2}+\mathrm{o}\left(E^{n / 2}\right), \quad \text { as } E \rightarrow \infty .
$$


This, and further research on additional terms in the asymptotic expansion led to the famous question of M. Kac: "Can one hear the shape of a drum?" 8 .

However, despite the appealing simplicity of this leading order asymptotics, neither compactness nor finite volume of $\Omega$ are necessary conditions for purely discrete spectrum of the Dirichlet Laplacian, denoted from now on by $-\Delta^{D}$. A class of 2-dimensional examples for infinite volume domains with discrete spectrum, as well as their leading order eigenvalue asymptotics was given by B. Simon in [12. He considered 2-dimensional domains of the form:

$$
\Omega_{\alpha}:=\left\{(x, y) \in \mathbb{R}^{2}:|x|^{\alpha}|y|<1\right\}
$$

with $\alpha>0$ and derived the asymptotics:

$$
N(E)=\left\{\begin{array}{l}
\zeta(\alpha)\left(\frac{\pi}{2}\right)^{-\alpha} \frac{\Gamma\left(\frac{1}{2} \alpha+1\right)}{\sqrt{\pi} \Gamma\left(\frac{1}{2} \alpha+\frac{3}{2}\right)} E^{\frac{\alpha+1}{2}}+o\left(E^{\frac{\alpha+1}{2}}\right), \quad \alpha>1 \\
\frac{1}{\pi} E \ln E+o(E \ln (E)), \quad \alpha=1,
\end{array}\right.
$$

where $\zeta$ denotes the Riemannian zeta function and $\Gamma$ denots the Gamma function. For $1>\alpha>0$ the first formula holds if one replaces $\alpha$ by $\alpha^{-1}$. This article is concerned with an extension of this example of eigenvalue asymptotics to higher dimensions. To this end we will determine the spectral asymptotics for (most members of) the class of Schrödinger operators given by:

$$
H_{n}^{\alpha}=-\Delta+\prod_{i=1}^{n}\left|x_{i}\right|^{\alpha_{i}}=-\Delta+V(x), \quad\left(x_{1}, \ldots, x_{n}\right) \in \mathbb{R}^{n}, \quad \alpha_{i} \in \mathbb{R}_{+}^{*} .
$$

If $n \neq 1$ their potentials are non-confining, yet their spectrum is purely discrete as we will see shortly. In turn this will enable us to find the eigenvalue asymptotics of the Dirichlet Laplacian $-\Delta^{D}$ on domains of the form:

$$
\Omega_{n}^{\alpha}=\left\{x \in \mathbb{R}^{n}: \prod_{j=1}^{n}\left|x_{j}\right|^{\alpha_{j} / \alpha_{n}}<1\right\} .
$$

Remark: Define the classical energy surfaces:

$$
\Sigma_{E}=\left\{(x, \xi) \in T^{*} \mathbb{R}^{n}:\|\xi\|^{2}+V(x)=E\right\},
$$

equipped with the (classical flow invariant) Liouville measures $\mathrm{dLVol}_{E}$, and the classical areas:

$$
A(E)=\bigcup_{e \leq E} \Sigma_{e}=\left\{(x, \xi) \in T^{*} \mathbb{R}^{n}:\|\xi\|^{2}+V(x) \leq e\right\},
$$

for the usual Lebesgue measure $d \mu$. Then, for any choice of the powers $\alpha_{i}$, we deal with non-compact energy surfaces of infinite volume, i.e. $\operatorname{Lvol}_{E}\left(\Sigma_{E}\right)=$ $+\infty$. Since we also have $\mu(A(E))=+\infty$ for $E>0$, Weyl's law does apply, neither in the classical nor the micro-local category. 
Despite this fact, B. Simon determined the asymptotics of the Schrödinger operators above in the case $n=2$ [12]. We will extend the calculation of these asymptotics to dimensions $n>2$ covering both the generic case $\alpha_{i} \neq \alpha_{j}, \forall i, j$ as well as the most singular case $\alpha_{i}=\alpha_{j} \forall i, j$. Up to a permutation of coordinates we can freely assume $\alpha_{1} \geq \cdots \geq \alpha_{n}>0$. Our main results then read as follows:

Theorem 1 For $n \geq 2$, assume $\alpha_{1}>\cdots>\alpha_{n}>0$ and define:

$$
d_{n}=d_{n}(\alpha):=\frac{\alpha_{1}+\ldots+\alpha_{n-1}+2}{2 \alpha_{n}} .
$$

Then, as $t$ tends to $0^{+}$, we have:

$$
\lim _{t \rightarrow 0^{+}} t^{\left(d_{n}+1 / 2\right)} \operatorname{Tr}\left(e^{-t H_{n}^{\alpha}}\right)=\frac{\operatorname{Tr}\left(\left(H_{n-1}^{\alpha}\right)^{-d_{n}}\right)}{\pi^{n / 2}} \Gamma\left(d_{n}+1\right),
$$

where:

$$
\operatorname{Tr}\left(\left(H_{n-1}^{\alpha}\right)^{-d_{n}}\right)=\operatorname{Tr}\left(\left(-\Delta_{x_{1} \ldots x_{n-1}}+\prod_{i=1}^{n-1}\left|x_{i}\right|^{\alpha_{i}}\right)^{-d_{n}}\right)<\infty,
$$

is the spectral-zeta function, evaluated at $d_{n}$, of the (n-1)-dimensional Schrödinger operator obtained by removing the direction of smallest decay at infinity.

Assuming that all exponents are equal we prove:

Theorem 2 If $\alpha_{1}=\cdots=\alpha_{n}=\alpha_{0}$, and thus $d_{n}=\frac{n-1}{2}+\alpha_{0}^{-1}$, then as $t$ tends to $0^{+}$we have:

$$
\lim _{t \rightarrow 0^{+}} t^{n / 2+\alpha_{0}^{-1}}|\ln (t)|^{-(n-1)} \operatorname{Tr}\left(e^{-t H_{n}^{\alpha}}\right)=\frac{\Gamma\left(1+\alpha_{0}^{-1}\right)\left(n / 2+\alpha_{0}^{-1}\right)^{(n-1)}}{\pi^{n / 2}(n-1) !} .
$$

Subsequently, using the Tauberian theorem of Karamata, cf. [12, we can prove that the eigenvalue counting functions $N_{H_{n}^{\alpha}}$ given by:

$$
N_{H_{n}^{\alpha}}(E)=\#\left\{j \in \mathbb{N}: \lambda_{j} \in \sigma\left(H_{n}^{\alpha}\right), \lambda_{j} \leq E\right\}
$$

satisfy the following asymptotic laws as $E \rightarrow \infty$ :

Theorem 3 For $n \geq 2$, and assuming $\alpha_{1}>\cdots>\alpha_{n}>0$ we have:

$$
\lim _{E \rightarrow \infty} E^{-\left(d_{n}+1 / 2\right)} N_{H_{n}^{\alpha}}(E)=\frac{\operatorname{Tr}\left(\left(H_{n-1}^{\alpha}\right)^{-d_{n}}\right) \Gamma\left(d_{n}+1\right)}{\pi^{n / 2} \Gamma\left(d_{n}+3 / 2\right)},
$$

where $d_{n}$ and $\operatorname{Tr}\left(\left(H_{n-1}^{\alpha}\right)^{-d_{n}}\right)$ are as in Theorem 1.

Theorem 4 If $\alpha_{1}=\cdots=\alpha_{n}=\alpha_{0}$, then we have:

$$
\lim _{E \rightarrow \infty} E^{-\left(n / 2+\alpha_{0}^{-1}\right)} \ln (E)^{-(n-1)} N_{H_{n}^{\alpha}}(E)=\frac{\Gamma\left(1+\alpha_{0}^{-1}\right)\left(n / 2+\alpha_{0}^{-1}\right)^{(n-1)}}{\Gamma\left(n / 2+\alpha_{0}^{-1}+1\right) \pi^{n / 2}(n-1) !} .
$$


Finally, this will imply the following result for the spectrum of the Dirichlet Laplacian $-\Delta^{D}$ on the domains $\Omega_{n}^{\alpha}$ :

Theorem 5 For $\alpha_{1}>\cdots>\alpha_{n}>0$ the Dirichlet-Laplacian $-\Delta^{D}=-\Delta_{\alpha, n}^{D}$ attached to the domain $\Omega_{n}^{\alpha}, n \geq 2$ has discrete spectrum and the counting function of eigenvalues satisfies:

$$
\lim _{E \rightarrow \infty} E^{-\left(q(\alpha)+\frac{1}{2}\right)} N_{-\Delta_{\alpha, n}^{D}}(E)=\frac{\operatorname{Tr}\left(\left(-\Delta_{\alpha, n-1}^{D}\right)^{-q(\alpha)}\right) \Gamma(q(\alpha)+1)}{\pi^{n / 2} \Gamma(q(\alpha)+3 / 2)} .
$$

where $-\Delta_{\alpha, n-1}^{D}$ is the Dirichlet Laplacian on the $n-1$-dimensional domain obtained by projecting $\Omega_{n}^{\alpha}$ onto the $x_{n}=0$ hyperplane and

$$
q(\alpha)=\frac{\alpha_{1}+\ldots+\alpha_{n-1}}{2 \alpha_{n}} .
$$

Theorem 6 The Dirichlet-Laplacian $-\Delta^{D}$ on $\Omega:=\left\{x \in \mathbb{R}^{n}\left|\prod_{i=1}^{n}\right| x_{i} \mid<1\right\}$, $n \geq 2$ has discrete spectrum and:

$$
\lim _{E \rightarrow \infty} \ln (E)^{-(n-1)} E^{-\frac{n}{2}} N_{-\Delta^{D}}(E)=\frac{n^{n-1}}{\Gamma\left(\frac{n}{2}\right) \sqrt{\pi^{n}} 2^{n-1}(n-1) !} .
$$

With regards to other work on similar operators, we first mention the work of D. Robert [9] on the family of potentials:

$$
V(x, y)=\left(1+x^{2}\right)^{r} y^{2 l}, \quad r, l \in \mathbb{R}^{+} .
$$

Using methods of microlocal analysis, he finds the leading order asymptotics for the corresponding Schrödinger operators. This precedes Simon's work by about two years and shows up the great difficulties to derive spectral estimates in presence of a non-confining potential.

Some extensions and alterations of the Simon example using the methods developed in his paper have also been published. In [1], Aramaki and Nurmuhammad have considered the potentials:

$$
V(z)=V(x, y)=\|x\|^{2 p}\|x\|^{2 q}, \quad p, q>0,
$$

and derived the leading order asymptotics for these cases. More recently, in [7. Exner and Barseghyan showed the discreteness of the spectrum, and obtained some bounds on moments of eigenvalues, for Schödinger operators with potentials unbounded from below:

$$
\left.|x y|^{p}-\lambda\left(x^{2}+y^{2}\right)^{\frac{p}{p+2}}, p \geq 1, \lambda \in\right] 0, \lambda_{\text {crit }}[.
$$


The main contribution of our work is to increase dimensions with even more separate factors in the definition of the potentials (resp. domains for the Dirichlet Laplacian) while keeping track of the top order coefficients of the asymptotic expansion of the counting function of eigenvalues.

The constant involved in these asymptotics, explicitely given as a spectral zetafunction of a lower dimensional operator, is of great interest and connects very much in line with the results of all the other work mentioned above.

This work is part of the second author's PhD thesis.

Before we begin our analysis we recall that for a Schrödinger operator with a positively homogeneous potential of degree $p \neq-2$, i.e. $V(t x)=|t|^{p} V(x)$ for all $x$, we have the following scaling relations for all $c>0$ :

$$
\begin{aligned}
& \sigma(-\Delta+c V)=c^{\frac{2}{p+2}} \sigma(-\Delta+V), \\
& \sigma(-c \Delta+V)=c^{\frac{p}{p+2}} \sigma(-\Delta+V) .
\end{aligned}
$$

These are equalities between spectra and these scaling relations are independent of the dimension $n$.

Following the strategy of B. Simon, who approached the two-dimensional case with what he calls the ' sliced-bread inequalities ', we recall now for the readers convenience the classical Tauberian theorem relating the small time behavior of the quantum partition function

$$
Z_{Q}(t)=\operatorname{Tr}\left(e^{-t H}\right)
$$

and the large energy behavior of the counting function $N_{H}(E)$ as seen in [12]:

Theorem 7 (Karamata's Tauberian Theorem.)

Let $H=-\Delta+V$ with $V$ continuous and non-negative. When $l$ and $d$ are positive, we have the equivalences:

$$
\begin{gathered}
\lim _{E \rightarrow+\infty} E^{-l} N_{H}(E)=c \Leftrightarrow \lim _{t \rightarrow 0^{+}} t^{l} \operatorname{Tr}\left(e^{-t H}\right)=c \Gamma(l+1), \\
\lim _{E \rightarrow+\infty} \frac{E^{-l}}{(\log E)^{d}} N_{H}(E)=c \Leftrightarrow \lim _{t \rightarrow 0^{+}} \frac{t^{l}}{|\log (t)|^{d}} \operatorname{Tr}\left(e^{-t H}\right)=c \Gamma(l+1) .
\end{gathered}
$$

So we will concentrate on the short time asymptotics of $Z_{Q}$ but the infinite volume of the energy surfaces provides a serious obstacle. As we remarked earlier, in the case of the operators we are interested in the common trick to estimate the quantum partition function by a classical integral fails for precisely this reason. Indeed if we define:

$$
Z_{c l}(t)=\frac{1}{(2 \pi)^{n}} \int_{T^{*} \mathbb{R}^{n}} e^{-t\left(\|\xi\|^{2}+V(x)\right)} d x d \xi,
$$


the inequality $Z_{Q}(t) \leq Z_{c l}(t)$ is valid (this can be viewed as a convexity property of the exponential function or a consequence of the abstract Golden-Thompson inequality), but for the product potential:

$$
V_{\alpha}(x):=\prod_{i=1}^{n}\left|x_{i}\right|^{\alpha_{i}}
$$

we get:

$$
\begin{gathered}
(2 \pi)^{n} Z_{c l}(t)=\left(\frac{\pi}{t}\right)^{\frac{n}{2}} \int_{\mathbb{R}^{n}} e^{-t V_{\alpha}(x)} d x \\
=\left(\frac{4 \pi}{t}\right)^{\frac{n}{2}} \Gamma\left(\frac{\alpha_{n}+1}{\alpha_{n}}\right) \int_{\mathbb{R}_{+}^{n-1}}\left(t \prod_{j=1}^{n-1}\left|x_{j}\right|^{\alpha_{j}}\right)^{-\frac{1}{\alpha_{n}}} d x_{1} \ldots d x_{n-1} \\
=\left(\frac{4 \pi}{t}\right)^{\frac{n}{2}} \Gamma\left(\frac{\alpha_{n}+1}{\alpha_{n}}\right) t^{\frac{1-n}{\alpha_{n}}} \prod_{j=1}^{n-1} \int_{0}^{\infty} x_{j}^{-\frac{\alpha_{j}}{\alpha_{n}}} d x_{j}=+\infty,
\end{gathered}
$$

none of these integrals being convergent on $[0, \infty]$, for any choice of the $\alpha_{j}$ (the convergence at the origin implying the divergence at infinity and vice-versa). Despite the fact that the classical estimate does not yield any useful information, we can easily obtain:

Lemma 8 For any $n$ and any $\alpha \in\left(\mathbb{R}_{+}^{*}\right)^{n}$, the spectrum of $H_{n}^{\alpha}$ is discrete.

Let us give first a rough proof that the $H_{n}^{\alpha}$ 's have discrete spectrum. This approach will not even catch the right asymptotic power. In dimension 2 , this was given by B. Simon in [11] as the most elementary proof between 6 different proofs of the discreteness of the spectrum.

Proof of Lemma 8, Using that the spectrum of the 1 dimensional operators:

$$
-\Delta+|x|^{\nu}, \nu>0
$$

has a strictly positive lowest eigenvalue $\lambda_{0}(\nu)$, by scaling (see Eq.(11) we get the following lower bound valid in the sense of quadratic forms on $H^{1}\left(\mathbb{R}^{n}\right)$ :

$$
-\Delta_{x_{1}, \cdots, x_{n}}+\left|x_{1}\right|^{\alpha_{1}} \prod_{j=2}^{n}\left|x_{j}\right|^{\alpha_{j}} \geq-\Delta_{x_{2}, \ldots, x_{n}}+\lambda_{0}\left(\alpha_{1}\right)\left(\prod_{j=2}^{n}\left|x_{j}\right|^{\alpha_{j}}\right)^{\frac{2}{2+\alpha_{1}}} .
$$

By induction, and symmetrization w.r.t. $x_{1}, \cdots, x_{n}$, it is easy to show that there exists $n$ positive continuous functions $f_{i}: \mathbb{R} \rightarrow \mathbb{R}_{+}$with $\lim _{t \rightarrow \pm \infty} f_{i}(t)=+\infty$ such that:

$$
-\Delta+\prod_{j=1}^{n}\left|x_{j}\right|^{\alpha_{j}} \geq \sum_{j=1}^{n}\left(-\Delta_{x_{j}}+f_{j}\left(x_{j}\right)\right)=\sum_{j=1}^{n} T_{j} .
$$


A fortiori, e.g. by a min-max argument1 1 , the spectrum of $H_{n}^{\alpha}$ is discrete each of the operators $T_{j}$ appearing in the r.h.s. being a 1-dimensional Schrödinger operator with confining potential. It is also easy to verify that $f_{j}(t) \geq C|t|^{\eta_{j}}$ for some $\eta_{j} \in \mathbb{Q}_{+}^{*}$.

Remark 9 As it was observed in the 2 dimensional case, the inequality:

$$
Z_{Q}(t)=\operatorname{Tr} e^{-t H_{n}^{\alpha}} \leq \prod_{j=1}^{n} \operatorname{Tr} e^{-t T_{j}}
$$

can be exploited to get an upper bound, e.g. using Bohr-Sommerfed quantization conditions for each $T_{j}$ (see [2], chapter 5). But these bounds are not good in view of the results stated in Theorem 2 and 3.

\section{Comments and perspectives:}

To our knowledge very few things are known about the interpretation of the results stated in Theorem 1 and 2 in terms of geometry, physics or dynamical systems. For example, a geometrical interpretation of the constants appearing in the short time expansion of $e^{-t H_{n}^{\alpha}}$ cannot have a 'classical' meaning (for the usual symplectic structure of the phase space $T^{*} \mathbb{R}^{n}$ ). At least these coefficients can be used to construct some (global) measures on eigenvectors:

$$
w(a)=\lim _{t \rightarrow 0^{+}} \frac{\operatorname{Tr} A e^{-t H_{n}^{\alpha}}}{\operatorname{Tr} e^{-t H_{n}^{\alpha}}}, A=\operatorname{Op}(a), a \in S^{0}\left(\mathbb{R}^{2 n}\right),
$$

i.e. the asymptotic results stated in theorems 1 and 2 provides some normalization factors (in terms of probability measures) for the statistical distribution of eigenfunctions in the phase space. Also, because of the strong singularities of the potential (and of the Liouville measure) on any hypersurface $\left\{x_{j}=0\right\}$, one could expect some concentration phenomena for the associated eigenfunctions estimates like it was observed in [4, 5].

Finally the approach based on the asymptotic behavior of the quantum partition function $Z_{Q}(t)$ does not allow to see very much concerning the classical dynamics generated by the potentials $V_{\alpha}$ (when the dynamics is globally defined in the usual way, meaning that the Hamiltonian vector field has the Lipschitz regularity). But, as a matter of honesty, the usual semi-classical methods and their underlying stationary-phase approximations seem to be inefficient because of the infinite volume of energy surfaces.

We will conclude the introduction with the proof of theorems 6 and 7. Of course, because of the scaling properties of our operators similar results are valid for Dirichlet-Laplacian in domains $\Omega_{\alpha}^{n}(a)=\left\{x \in \mathbb{R}^{n}:\left|x_{1}\right|^{\alpha_{1}} \cdots\left|x_{n}\right|^{\alpha_{n}}<a\right\}$ for any positive $a$.

\footnotetext{
${ }^{1}$ One could also exponentiate the functional inequality and take the trace.
} 
Proof of theorem 6 and 7: The proof of these results is straightforward if we use a sequence of potentials with strictly increasing exponents $(\alpha)_{j}=j . \alpha=$ $\left(j \alpha_{1}, \cdots, j \alpha_{n}\right), j>0$. The ratios of theorem 1 satisfy:

$$
d_{n}\left((\alpha)_{j}\right)=\frac{j \alpha_{1}+\ldots+j \alpha_{n-1}+2}{2 j \alpha_{n}} \rightarrow \frac{\alpha_{1}+\ldots+\alpha_{n-1}}{2 \alpha_{n}} \text { as } j \rightarrow+\infty .
$$

On the other side, if $j$ tends to infinity by homogenity we have:

$$
V_{(\alpha)_{j}}=\left(V_{\alpha}\right)^{j} \rightarrow\left\{\begin{array}{c}
0 \text { if } V_{\alpha}<1 \\
1 \text { if } V_{\alpha}=1 \\
+\infty \text { if } V_{\alpha}>1
\end{array}\right.
$$

By taking the exponential (the potentials are everywhere positive) we get the desired result since $e^{-t\left(-\Delta+V_{\left.(\alpha)_{j}\right)}\right.}$ converges to $e^{-t\left(-\Delta_{n, \alpha}^{D}\right)}$ as $j \rightarrow \infty$ in the trace norm.

The rest of the paper is organized as follows: in section 2 we recall the sliced bread estimate used to get good upper bound on $Z_{Q}(t)$. Section 3 then contains the proof of Theorem 2 , while in section 4 we prove the technically more involved asymptotics of Theorem 3 .

\section{Slicing techniques for the partition function:}

As was noted in the last section, the Tauberian theorem of Karamata allows us to focus on the small time divergence of the partition function $Z_{Q}(t)$. When analyzing the trace of an operator of this type, it is useful to 'slice' the problem. That is, we write an operator $A=-\Delta+V(x, y),(x, y) \in \mathbb{R}^{a} \times \mathbb{R}^{b}=\mathbb{R}^{c}$ on $L^{2}\left(\mathbb{R}^{c}\right)$, with $V(x, y)$ continuous and bounded from below as a sum:

$$
A=-\Delta_{x}+A_{x}
$$

Here $A_{x}=-\Delta_{y}+V(x, y)$ as an operator on $L^{2}\left(\mathbb{R}^{b}\right)$ depending on $x$. Let $\lambda_{k}(x)$ be the increasing sequence of eigenvalues of $A_{x}$, repeated according to their multiplicities. Define:

$$
\begin{gathered}
Z_{S B}(t)=\sum_{k} \operatorname{Tr}_{L^{2}\left(\mathbb{R}^{a}\right)}\left(e^{-t\left(\Delta_{x}+\lambda_{k}(x)\right)}\right), \\
Z_{S G T}(t)=\int \frac{e^{-t\|\xi\|^{2}}}{(2 \pi)^{a}} \operatorname{Tr}_{L^{2}\left(\mathbb{R}^{b}\right)}\left(e^{-t\left(A_{x}\right)}\right) d^{a} \xi d^{a} x, \\
Z_{c l}(t)=\int \frac{e^{-t\left(\|\xi\|^{2}+V(x, y)\right)}}{(2 \pi)^{c}} d^{c} \xi d^{a} x d^{b} y .
\end{gathered}
$$

Here SB stands for sliced bread, SGT is sliced Golden-Thompson. The 'slicedbread' and 'sliced-Golden-Thompson' techniques are now centered around the following theorem, which is due to B. Simon [12]: 
Theorem 10 (Barry Simon's sliced bread inequalities.)

For each $t>0$ we have:

$$
Z_{Q}(t) \leq Z_{S B}(t) \leq Z_{S G T}(t) \leq Z_{c l}(t) .
$$

The potentials we deal with in this paper provide examples where $Z_{c l}(t)=\infty$ and, depending on the choice of $\alpha_{i}$, even $Z_{S G T}(t)=\infty$, yet the traces $Z_{S B}(t)$ and $Z_{Q}(t)$ exist. This provides a set of examples where these estimates prove to be more powerful than the classical one. In the case of the potentials covered in this paper, working with either $Z_{S G T}(t)$ or $Z_{S B}(t)$ leads to studying 'partial trace' functions of the type:

$$
F\left(x_{n}, t\right)=\operatorname{Tr}_{L^{2}\left(d x_{1} \ldots d x_{n-1}\right)}\left(\exp \left[-t\left(\Delta_{x_{1} \ldots x_{n-1}}+\prod_{i=1}^{n}\left|x_{i}\right|^{\alpha_{i}}\right)\right]\right)
$$

This function satisfies a remarkable functional equation:

Lemma 11 The function $F\left(x_{n}, t\right)$ defined above satisfies the following scaling relation:

$$
F\left(x_{n}, t\right)=F\left(x_{n} t^{d_{n}}, 1\right)=F\left(1, t\left|x_{n}\right|^{1 / d_{n}}\right) .
$$

Proof. The homogeneity of the potential is crucial in proving this. Use the scaling relations of Eqs.(12) and thus show:

$$
\begin{aligned}
& F\left(x_{n}, t\right)=\operatorname{Tr}_{L^{2}\left(d x_{1} \ldots d x_{n-1}\right)}\left(\exp \left[-t\left(-\Delta_{x_{1} \ldots x_{n-1}}+\prod_{i=1}^{n}\left|x_{i}\right|^{\alpha_{i}}\right)\right]\right) \\
& =\operatorname{Tr}_{L^{2}\left(d x_{1} \ldots d x_{n-1}\right)}\left(\exp \left[-\left(-\Delta_{x_{1} \ldots x_{n-1}}+\left|t^{d_{n}} x_{n}\right|^{\alpha_{n}} \prod_{i=1}^{n-1}\left|x_{i}\right|^{\alpha_{i}}\right)\right]\right) \\
& =\operatorname{Tr}_{L^{2}\left(d x_{1} \ldots d x_{n-1}\right)}\left(\exp \left[-t\left|x_{n}\right|^{\left(1 / d_{n}\right)}\left(-\Delta_{x_{1} \ldots x_{n-1}}+\prod_{i=1}^{n-1}\left|x_{i}\right|^{\alpha_{i}}\right)\right]\right) .
\end{aligned}
$$

For the first equality set $c=t$ and apply (1) then (2), for the second one set $c=\left|x_{n}\right|$ and work in reverse order.

\section{$3 \quad$ Eigenvalue asymptotics, $\alpha_{i} \neq \alpha_{j}, \quad \forall i \neq j$}

This case is techniqually less involved due to the finiteness of $Z_{S G T}(t)$. When all indices are different, up to permutation of coordinates we can assume that:

$$
V_{\alpha}(x)=\prod_{i=1}^{n}\left|x_{i}\right|^{\alpha_{i}}, \text { with } \alpha_{1}>\ldots>\alpha_{n} .
$$

The proof will be by induction over dimension. The dimension 2 case was shown by B. Simon in [12]. Suppose now that in dimension $n-1$, we have:

$$
\lim _{t \rightarrow 0} t^{\left(d_{n-1}+1 / 2\right)} \operatorname{Tr}\left(e^{-t H_{n-1}^{\alpha}}\right)=\operatorname{Tr}\left(\left(H_{n-2}^{\alpha}\right)^{-d_{n-1}}\right) \pi^{-1 / 2} \Gamma\left(d_{n-1}+1\right) .
$$


We will estimate $Z_{Q}(t)$ from above and below, and then both show that the difference of these bounds asymptotically goes to zero and compute the asymptotics of the upper bound. The lower bound will be found using the FeynmanKac formula that gives a representation of the trace $Z_{Q}(t)$ of the heat kernel as an expectation value of Brownian motion running for time $2 t$. The upper bound will be found using the sliced Golden-Thompson trace. We will slice in direction of the coordinate of smallest power in the potential, $x_{n}$.

Remark 12 The slicing for $Z_{S G T}(t)$ works only if one takes slices in the right direction, that is, the one with smallest exponent in the potential. As we will see one has no choice, as the integrals will not converge if one slices in a different way.

To start, we note that from the sliced bread inequalities, we know already that: $Z_{Q}(t) \leq Z_{S G T}(t)$. Let us first rewrite this upper bound a little. Doing the $\xi$-integral explicitly, we get:

$$
Z_{S G T}(t)=(\pi t)^{-1 / 2} \int_{0}^{\infty} F\left(x_{n}, t\right) d x_{n} .
$$

Lower bound: Next we will prove a lower bound that is easy to compare to the expression of $Z_{S G T}$ as an integral of $F$. Using the Feynman-Kac formula we rewrite $Z_{Q}(t)$ just like Barry Simon did in the two dimensional case, see [10, 12]:

$Z_{Q}(t)=(4 \pi t)^{-n / 2} \int_{x \in \mathbb{R}^{n}} \mathbb{E}_{x, x ; 2 t}\left[\exp \left(-\int_{0}^{2 t} \frac{1}{2}\left|b_{1}(s)\right|^{\alpha_{1}} \ldots\left|b_{n-1}(s)\right|^{\alpha_{n-1}}\left|b_{n}(s)\right|^{\alpha_{n}} d s\right)\right] d x$,

where $b_{i}$ denotes the 1-dimensional Brownian motion and $\mathbb{E}_{x, x ; 2 t}$ is the conditional expectation value w.r.t. the Brownian motion with conditions to start and end 2 in $x$ in time $2 t$. We proceed by cutting off all paths such that:

$$
\sup _{0 \leq s \leq 2 t}\left|b_{n}(s)-x_{n}\right|>1,
$$

and replacing $\left|b_{n}(s)\right|^{\alpha_{n}}$ by its upper bound $\left(\left|x_{n}\right|+1\right)^{\alpha_{n}}$. Using that our potential is a product function, and since the probability measure of the $n$-dimensional Brownian motion is a product measure, this gives a lower bound for $Z_{Q}(t)$, namely:

$$
\begin{aligned}
Z_{Q}(t) & \geq(\pi t)^{-\frac{1}{2}}(1-\rho(t)) \int_{0}^{\infty} F\left(\left|x_{n}\right|+1, t\right) d x_{n} \\
& =(\pi t)^{-\frac{1}{2}}(1-\rho(t)) \int_{1}^{\infty} F\left(x_{n}, t\right) d x_{n} .
\end{aligned}
$$

\footnotetext{
${ }^{2}$ Of course, the fact that $\mathbb{E}_{x, y ; 2 t}$ is restricted to the diagonal $\{x=y\}$ corresponds to the fact that $Z_{Q}$ is the trace of the heat kernel.
} 
This inequality is valid by symmetry and by the following two facts. First, the probability that a path leaves a compact interval $\left[x_{n}-1, x_{n}+1\right]$ during a small time interval $[0,2 t]$ is small:

$$
\rho(t) \geq \operatorname{Prob} \sup _{0 \leq s \leq 2 t}\left(\left|b_{n}(s)-x_{n}\right|>1\right)
$$

with $\rho(t) \rightarrow 0$ as $t \rightarrow 0^{+}$. In fact, a classical result concerning the 1 -dimensional Brownian motion is that for each $\varepsilon>0$, there exists a positive constant $C(\varepsilon)$ such that:

$$
\rho(t) \leq C(\varepsilon) e^{-(1-\varepsilon) / 4 t}, \text { as } t \rightarrow 0^{+} .
$$

Second, the monotony of the exponential, the positivity of the integral and the product structure of the potential gives a lower bound at $\left|x_{n}\right|+1$.

Upper bound: Let us now analyze the upper bound more closely. Theorem 2 follows from the following three statements:

$$
\begin{gathered}
\lim _{t \rightarrow 0} t^{d_{n}} \int_{0}^{1} F\left(x_{n}, t\right) d x_{n}=0 . \\
\lim _{t \rightarrow 0} t^{d_{n}} \int_{0}^{\infty} F\left(x_{n}, t\right) d x_{n}=\operatorname{Tr}\left(\left(H_{n-1}^{\alpha}\right)^{-d_{n}}\right) \Gamma\left(d_{n}+1\right) . \\
\operatorname{Tr}\left(\left(H_{n-1}^{\alpha}\right)^{-d_{n}}\right)<\infty .
\end{gathered}
$$

This will finish the proof because equation (3) implies that our upper bound and lower bound agree asymptotically, while equations (415) prove the asymptotic power and constant. We treat now the result stated in equation (3). According to the hypothesis of induction we have:

$$
F(1, t) \sim C t^{-\frac{1}{2}-d_{n-1}}, \text { as } t \rightarrow 0^{+}
$$

for some $C>0$. It follows that for $|x|$ in a compact subset of $\mathbb{R}_{+}$and $t>0$ small:

$$
F(x, t) \leq \tilde{C}\left(|x|^{\frac{1}{d_{n}}}\right)^{-\frac{1}{2}-d_{n-1}} t^{-\frac{1}{2}-d_{n-1}} .
$$

Hence since:

$$
\begin{gathered}
d_{n}-\frac{1}{2}-d_{n-1}=\frac{\alpha_{1}+\cdots+\alpha_{n-1}+2}{2 \alpha_{n}}-\frac{1}{2}-\frac{\alpha_{1}+\cdots+\alpha_{n-2}+2}{2 \alpha_{n-1}} \\
=\frac{\alpha_{1}+\cdots+\alpha_{n-1}+2}{2 \alpha_{n}}-\frac{\alpha_{1}+\cdots+\alpha_{n-1}+2}{2 \alpha_{n-1}}>0 .
\end{gathered}
$$

The decay w.r.t. $t$ is achieved. It remains to show that the integral w.r.t. $x$ is finite. For $t$ small we have that:

$$
\left.\int_{0}^{1} F(x, t) d x \leq C(t) \int_{0}^{1}|x|^{\frac{1}{d_{n}}}\right)^{-\frac{1}{2}-d_{n-1}} d x .
$$


We see that this singularity in $x=0$ is integrable if and only if:

$$
-\frac{1}{d_{n}}\left(\frac{1}{2}+d_{n-1}\right)>-1
$$

since we have:

$$
\frac{1}{d_{n}}\left(\frac{1}{2}+d_{n-1}\right)=\frac{2 \alpha_{n}}{\alpha_{1}+\cdots+\alpha_{n-1}+2} \frac{\alpha_{1}+\cdots+\alpha_{n-1}+2}{2 \alpha_{n-1}}=\frac{\alpha_{n}}{\alpha_{n-1}}<1,
$$

we obtain that the integral is finite.

Remark 13 This result concerning the singularity in $x=0$ is asymptotically exact as $t \rightarrow 0^{+}$so that the condition $\alpha_{n}<\alpha_{n-1}$ is necessary to get Eq.(3). Hence, like in dimension 2 , there is a good way to slice imposed by the exponents of the potential. One slices always in direction of the smallest exponent. Also sliced Gordon Thompson is not working with $\alpha_{n}=\alpha_{n-1}$ (log singularity at the origin) and sliced bread is required.

Let us now show the result stated in equation (44). First, via the functional equation for $F$, we can scale $t$ out as:

$$
\int_{0}^{\infty} F\left(x_{n}, t\right) d x_{n}=\int_{0}^{\infty} F\left(x_{n} t^{d_{n}}, 1\right) d x_{n}=t^{-d_{n}} \int_{0}^{\infty} F\left(x_{n}, 1\right) d x_{n} .
$$

Using again lemma 11, we can scale out the $n-1$ dimensional operator $H_{n-1}^{\alpha}$ via:

$$
\begin{gathered}
\int_{0}^{\infty} F\left(x_{n}, 1\right) d x_{n}=\int_{0}^{\infty} F\left(1, x_{n}^{1 / d_{n}}\right) d x_{n} \\
=d_{n} \int_{0}^{\infty} s^{l_{n}-1} F(1, s) d s=d_{n} \operatorname{Tr}\left(\int_{0}^{\infty} s^{d_{n}-1} e^{-s H_{n-1}^{\alpha}} d s\right) \\
=d_{n} \Gamma\left(d_{n}\right) \operatorname{Tr}\left(\left(H_{n-1}^{\alpha}\right)^{-d_{n}}\right)=\Gamma\left(d_{n}+1\right) \operatorname{Tr}\left(\left(H_{n-1}^{\alpha}\right)^{-d_{n}}\right),
\end{gathered}
$$

as required.

Finally, to achieve the proof of Theorem 1 it remains to establish the finiteness of $\operatorname{Tr}\left(\left(H_{n-1}^{\alpha}\right)^{-d_{n}}\right)$, stated in equation (5). As shown above, we have:

$$
\operatorname{Tr}\left(\left(H_{n-1}^{\alpha}\right)^{-d_{n}}\right)=\frac{1}{\Gamma\left(d_{n}+1\right)} \int_{0}^{\infty} F\left(1, x_{n}^{1 / d_{n}}\right) d x_{n} .
$$

We now show that the right hand side is finite. As we just have seen, for small $x_{n}$, the induction hypothesis tells us that:

$$
F\left(1, x_{n}^{1 / d_{n}}\right) \sim C x_{n}^{\frac{1}{d_{n}}\left(-\frac{1}{2}-d_{n-1}\right)}=C x_{n}^{-\frac{a_{n}}{a_{n}-1}}, \text { as } x_{n} \rightarrow 0^{+} .
$$


Since $\frac{a_{n}}{a_{n-1}}<1$, the singularity at zero is integrable. On the other hand, for $x_{n}$ large we see from the induction hypothesis that $F\left(1, x_{n}^{1 / d_{n}}\right)$ decays as $e^{-c x_{n}^{1 / d_{n}}}$ and it follows that $\operatorname{Tr}\left(\left(H_{n-1}^{\alpha}\right)^{-d_{n}}\right)<\infty$.

\section{Eigenvalue asymptotics, $\alpha_{1}=\cdots=\alpha_{n}=\alpha_{0}$.}

Consider now the singular case of equal exponents in all directions, that is:

$$
H_{n}^{\alpha}=-\Delta+\prod_{i=1}^{n}\left|x_{i}\right|^{\alpha_{0}}, \quad\left(x_{1}, \ldots, x_{n}\right) \in \mathbb{R}^{n}, \quad \alpha_{0} \in \mathbb{R}_{+}^{*} .
$$

We will prove the asymptotics of $Z_{Q}(t)=\operatorname{Tr}\left(e^{t H_{n}^{\alpha}}\right)$ again based on an induction in dimension argument. The structure of the proof is once more to find an upper and lower bound for $Z_{Q}(t)$ that asymptotically agree to first order. We have seen in the last section that $Z_{S G T}(t)=\infty$ in this situation and therefore, we will need to exploit the final inequality $Z_{Q}(t) \leq Z_{S B}(t)$ to get a finite upper bound. We will employ the Feynman-Kac formula to find a lower bound for $Z_{Q}(t)$. To begin with, we recall the claimed asymptotics:

$$
\lim _{t \rightarrow 0^{+}} t^{(n / 2)+\alpha_{0}^{-1}} \ln \left(t^{-1}\right)^{n-1} Z_{Q}(t)(E)=\frac{\Gamma\left(1+\alpha_{0}^{-1}\right)\left(n / 2+\alpha_{0}^{-1}\right)^{(n-1)}}{\pi^{n / 2}(n-1) !} .
$$

According to the Tauberian theorem of Karamata this imply:

$$
\lim _{E \rightarrow \infty} E^{-(n / 2)+\alpha_{0}^{-1}} \ln (E)^{-n-1} N_{H_{n}^{\alpha}}(E)=\frac{\Gamma\left(1+\alpha_{0}^{-1}\right)\left(n / 2+\alpha_{0}^{-1}\right)^{(n-1)}}{\Gamma\left(n / 2+\alpha_{0}^{-1}\right) \pi^{n / 2}(n-1) !}=: a_{n} .
$$

We proceed now with the computation of the asymptotics of the aforementioned bounds, starting with the upper one:

Upper bound. From the sliced bread inequalities, we know $Z_{Q}(t) \leq Z_{S B}(t)$. We compute now the asymptotics of $Z_{S B}$ directly, making heavy use of scaling arguments. To start, we prove some auxiliary results in the following lemma:

Lemma 14 Let $A_{g}=-\Delta_{x_{n}}+g\left|x_{n}\right|^{\gamma}, \gamma>0$. Let $F_{g}^{(\gamma)}(t)=\operatorname{Tr}\left(\exp \left(-t A_{g}\right)\right)$ and $N_{A_{g}}$ be the associated counting function. Also, denote by $N_{r}:=N_{B_{r}}$ the eigenvalue counting function of $B_{r}:=-\Delta_{x_{1}, \ldots, x_{n-1}}+r \prod_{i}^{n-1}\left|x_{i}\right|^{\alpha_{0}}$. Omit the indices $g, r$ whenever $r=1, g=1$. Then:

a) $F_{g}^{(\gamma)}(t)=F^{(\gamma)}\left(g^{\tau} t\right)$, where $\tau=2 /(\gamma+2)$,

b) $N_{r}(E)=N\left(r^{\eta} E\right)$, where $\eta=\left((n-1) \alpha_{0}+2\right) / 2$,

c) $\lim _{t \rightarrow 0^{+}} t^{\mu} F^{(\gamma)}(t)=\pi^{-1 / 2} \Gamma\left(1+\gamma^{-1}\right)$, where $\mu=(\gamma+2) / 2 \gamma$, 
d) $\lim _{t \rightarrow 0^{+}} t^{\mu+1} F^{\prime(\gamma)}(t)=-\mu \pi^{-1 / 2} \Gamma\left(1+\gamma^{-1}\right)$.

Proof: Part a) and b) follow by the scaling relations (1) and (2). Part c) follows from the observation that since for the operator $A_{g}$ in one dimension the potential $\left|x_{n}\right|^{\gamma}$ is confining, we actually have $\frac{Z_{c l}(t)}{F^{(\gamma)}(t)} \rightarrow 1$ as $t \rightarrow 0^{+}$, cf. [10. Now:

$$
Z_{c l}(t)=\frac{1}{2 \pi} \int_{\mathbb{R}^{2}} e^{-t\left(\xi^{2}+|x|^{\gamma}\right)} d x d \xi=t^{-\gamma} \pi^{-1 / 2} \Gamma\left(1+\gamma^{-1}\right),
$$

and the claim follows. Finally, part d) which tells us that the asymptotics of the differential of $F$ are given as the differential of the leading term of $F$ is shown by the following calculation:

$$
\begin{aligned}
-F^{\prime(\gamma)}(t) & =\int_{0}^{\infty} x e^{-t x} d N_{A}(x) \\
& =\int_{0}^{\infty} e^{-t x}(x t-1) N_{A}(x) d x \\
& =t^{-1} \int_{0}^{\infty} e^{-y}(y-1) N_{A}\left(\frac{y}{t}\right) d y .
\end{aligned}
$$

To conclude that the claimed asymptotics of $F^{\prime(\gamma)}$ hold we use the Karamata Tauberian theorem for $N_{A}$ and apply result $c$ ).

With the help of this lemma, we first rescale the sliced bread trace $Z_{S B}(t)$ and then represent it as an integral expression which is suitable for the computation of the asymptotics. Since $N_{r}$ is, up to the factor $r$ in the potential, the counting function of the operator $B_{1}=H_{n-1}^{\alpha}$ in dimension $n-1$, this will get us in position to use the induction hypothesis.

For the rescaling, let $\epsilon_{j}\left(x_{n}\right)$ be the $j$ th eigenvalue of $-\Delta_{x_{1}, \ldots, x_{n-1}}+\left|x_{n}\right|^{\alpha_{0}} \prod_{i=1}^{n-1}\left|x_{i}\right|^{\alpha_{0}}$ so with $d_{n}=\frac{n-1}{2}+\alpha_{0}^{-1}$ we get by relation $\mathrm{b}$ ):

$$
\epsilon_{j}\left(x_{n}\right)=\left|x_{n}\right|^{1 / d_{n}} \epsilon_{j}(1)=:|x|^{1 / d_{n}} \epsilon_{j} .
$$

Now compute:

$$
\begin{aligned}
Z_{S B}(t) & =\sum_{j} \operatorname{Tr}\left(\exp \left[-t\left(\Delta_{x}+\epsilon_{j}\left(x_{n}\right)\right)\right]\right) \\
& =\sum_{j} \operatorname{Tr}\left(\exp \left[-t\left(\Delta_{x}+\epsilon_{j}\left|x_{n}\right|^{1 / d_{n}}\right)\right]\right) \\
& =\sum_{j} \operatorname{Tr}\left(\exp \left[-t \epsilon_{j}^{b_{n}}\left(\Delta_{x}+\left|x_{n}\right|^{1 / d_{n}}\right)\right]\right) .
\end{aligned}
$$


where we have used scaling relation b) so: $b_{n}=\frac{d_{n}}{d_{n}+(1 / 2)}$. We now represent $Z_{S B}$ as an integral and use integration by parts:

$$
\begin{aligned}
Z_{S B}(t) & =\sum_{j} F^{\left(1 / d_{n}\right)}\left(t \epsilon_{j}^{b_{n}}\right) \\
& =\int_{0}^{\infty} F^{\left(1 / d_{n}\right)}\left(t E^{b_{n}}\right) d N(E) \\
& =-\int_{0}^{\infty} t b E^{b_{n}-1} F^{\prime\left(1 / d_{n}\right)}\left(t E^{b_{n}}\right) N(E) d E .
\end{aligned}
$$

There are no boundary terms for the following reasons:

First, we have $N=0$ for small $E>0$ since a direct evaluation shows that 0 is no eigenvalue of $H_{n-1}^{\alpha}$ and the spectrum of this operator is discrete by the hypothesis of induction.

Second, $F^{\left(1 / d_{n}\right)} N \rightarrow 0$ for fixed $t$ and $E \rightarrow \infty$, since for large energies, the trace $F^{\left(1 / d_{n}\right)}$ decays exponentially.

The rescaling led to $t E^{b_{n}}$ as the argument of $F^{\prime\left(1 / d_{n}\right)}$. This will prove to be crucial for the precise asymptotic analysis. We proceed from here very similarly to Simon's arguments for dimension 2 by analyzing this integral on several pieces. As we just argued, since $N(E)=0$ for $E>0$ small by our hypothesis, it is sufficient to integrate from the ground state energy $E_{0}>0$ to $\infty$. Now we pick values $E_{0}<E_{1}<E_{2}<\infty$ such that:

$$
E_{1}^{b_{n}} t=|\ln t|^{-1}, \quad E_{2}^{b_{n}} t=1 .
$$

We will now estimate the integral on $\left(E_{0}, E_{1}\right),\left(E_{1}, E_{2}\right),\left(E_{2}, \infty\right)$ separately and see that only the integral on $\left(E_{0}, E_{1}\right)$ will contribute to the top order coefficients.

$\left(E_{2}, \infty\right)$ : On this piece we first note that $N(E) \leq c E^{d_{n}} \ln (E)^{n-2}$ for all $E$ because of the hypothesis of induction, and in addition, in the region $y \geq 1$, $-F^{\prime\left(1 / d_{n}\right)}(y)=\sum \tilde{\epsilon}_{j} e^{-y \tilde{\epsilon}_{j}} \leq D e^{-c y}$, with $D, C>0$ and $\tilde{\epsilon}_{j}$ being the eigenvalues of $\Delta_{x_{n}}+|y|^{1 / d_{n}}$. Thus we estimate:

$$
\begin{aligned}
& -\int_{E_{2}}^{\infty}\left(t b_{n}\right) E^{b_{n}-1} N(E) F^{\left(1 / d_{n}\right)}\left(t E^{b_{n}}\right) d E \\
\leq & c_{1} \int_{E_{2}}^{\infty} t E^{b_{n}-1+d_{n}} \ln (E)^{n-2} \exp \left(-c t E^{b_{n}}\right) d E \\
= & c_{2} t^{-b_{n} / d_{n}} \int_{1}^{\infty}\left(\ln \frac{y}{t}\right)^{n-2} y^{d_{n} / b_{n}} e^{-c y} d y,
\end{aligned}
$$

for some constants $c_{1}, c_{2}$. We see that all terms in the integral are bounded by $t^{-d_{n} / b_{n}} \ln \left(t^{-1}\right)^{-(n-2)}=t^{-\left(d_{n}+1 / 2\right)} \ln \left(t^{-1}\right)^{-(n-2)}$ which is small on the 
level of $t^{-\left(d_{n}+1 / 2\right)} \ln \left(t^{-1}\right)^{-(n-1)}$.

$\left(E_{1}, E_{2}\right)$ : We bound $N(E)$ as before but use now $-F^{\prime\left(1 / d_{n}\right)}(y) \leq C y^{-d_{n}-\frac{3}{2}}$, by result d) of the previous lemma. Then, where $\mathrm{c}$ is a constant which changes from equation to equation:

$$
\begin{aligned}
& -\int_{E_{1}}^{E_{2}}\left(t b_{n}\right) E^{b_{n}-1} N(E) F^{\prime\left(1 / d_{n}\right)}\left(t E^{b_{n}}\right) d E \\
\leq & c \int_{E_{1}}^{E_{2}} t E^{b_{n}-1+d_{n}} \ln (E)^{n-2}\left(t E^{b_{n}}\right)^{-d_{n}-3 / 2} d E \\
\leq & c t^{-d_{n}-1 / 2} \int_{E_{1}}^{E_{2}} \ln (E)^{n-2} E^{-1} d E \\
\leq & c t^{-d_{n}-1 / 2} \ln \left(E_{2} / E_{2}\right)^{n-1},
\end{aligned}
$$

where we have used $b_{n}-1+d_{n}-b_{n}\left(d_{n}+\frac{3}{2}\right)=-1$, since $b_{n}\left(d_{n}+\frac{1}{2}\right)=d_{n}$. Because $\ln \left(E_{2} / E_{1}\right)=b_{n}^{-1} \ln (|\ln t|)$, this integral is small compared to $t^{-\left(d_{n}+1 / 2\right)} \ln \left(t^{-1}\right)^{-(n-1)}$.

$\left(E_{0}, E_{1}\right)$ : Finally, for the last piece we replace $F^{\prime\left(1 / d_{n}\right)}$ by its asymptotic value making a multiplicative error of the form $1+o(1)$. In other words, we can bound $F^{\prime}$ from above and below by:

$$
-(1 \pm \epsilon(t))\left(d_{n}+\frac{1}{2}\right) \pi^{-1 / 2} \Gamma\left(1+d_{n}\right)\left(t E^{b_{n}}\right)^{\left(d_{n}+3 / 2\right)},
$$

with $\epsilon(t) \rightarrow 0$. This is true because the argument of $F^{\prime}$ namely $t E^{b_{n}}$ is now bounded from above by $|\ln (t)|^{-1}$, which implies:

$$
\left|F^{\prime\left(1 / d_{n}\right)}\left(t E^{b_{n}}\right)-\left(d_{n}+\frac{1}{2}\right) \pi^{-1 / 2} \Gamma\left(1+d_{n}\right)\left(t E^{b_{n}}\right)^{\left(d_{n}+3 / 2\right)}\right|=o\left(\frac{1}{|\ln (t)|}\right) .
$$

Thus, if $\sim$ means the ratio goes to 1 , we see that:

$$
\begin{aligned}
A(t):= & -\int_{E_{0}}^{E_{1}}\left(t b_{n}\right) E^{b_{n}-1} N(E) F^{\prime\left(1 / d_{n}^{\prime}\right)}\left(t E^{b_{n}}\right) d E \\
\sim & \int_{E_{0}}^{E_{1}} t b_{n} E^{-1} \ln (E)^{n-2} t^{-d_{n}-3 / 2}\left(d_{n}+1 / 2\right) \pi^{-1 / 2} \Gamma\left(1+d_{n}\right) a_{n-1} \\
& {\left[\frac{N(E)}{a_{n-1} E^{d_{n}} \ln (E)^{n-2}}\right] d E, }
\end{aligned}
$$

where $a_{n-1}$ is just the constant of the eigenvalue counting function in dimension $(n-1)$. Now observe that as $t \rightarrow 0^{+}$the value of $E_{1}$ tends to $\infty$, while $E_{0}$ remains constant. Since by the induction hypothesis we also have:

$$
\frac{N(E)}{a_{n-1} E^{d_{n}} \ln (E)^{n-2}} \rightarrow 1 \quad \text { as } E \rightarrow \infty,
$$


we see now that we have another asymptotic equivalence, namely:

$$
\begin{aligned}
A(t) & \sim a_{n-1} b_{n}\left(d_{n}+1 / 2\right) \pi^{-1 / 2} \Gamma\left(1+d_{n}\right) t^{-d_{n}-1 / 2} \int_{E_{0}}^{E_{1}} E^{-1} \ln (E)^{n-2} d E \\
& =\frac{a_{n-1} d_{n} \Gamma\left(1+d_{n}\right)}{\pi^{1 / 2}} t^{-d_{n}-1 / 2} \frac{\ln \left(E_{1} / E_{0}\right)^{n-1}}{(n-1)},
\end{aligned}
$$

Now we compute:

$$
\ln \left(E_{1} / E_{0}\right)=\ln \left[c t^{-1 / b_{n}}|\ln t|^{-1 / b_{n}}\right] \sim \frac{1}{b_{n}} \ln \left(t^{-1}\right) .
$$

where $c$ is once more some constant. We conclude that:

$$
\begin{aligned}
A(t) t^{-d_{n}-1 / 2} \ln \left(t^{-1}\right)^{-(n-1)} & \sim \pi^{-1 / 2} a_{n-1} d_{n} b_{n}^{-(n-1)} \Gamma\left(1+d_{n}\right)(n-1)^{-1} \\
& =\frac{\Gamma\left(d_{3}\right)\left(d_{n+1}\right)^{n-1}}{\pi^{n / 2}(n-1) !} .
\end{aligned}
$$

So we get the claimed asymptotics for the upper bound:

$$
\lim _{t \rightarrow 0} t^{n / 2+\alpha_{0}^{-1}} \ln \left(t^{-1}\right)^{-(n-1)} \operatorname{Tr}\left(e^{-t H_{n}^{\alpha}}\right) \leq \frac{\Gamma\left(1+\alpha_{0}^{-1}\right)\left(n / 2+\alpha_{0}^{-1}\right)^{n-1}}{\pi^{n / 2}(n-1) !} .
$$

Lower bound. For the lower bound we will employ the Feynman-Kac formula again. Before we do so we prove the following lemma:

Lemma 15 Let $f: \mathbb{R} \rightarrow \mathbb{R}$ be any integrable function. Let $P: \mathbb{R}^{n} \rightarrow \mathbb{R}$ denote the map given by $P(x)=\prod_{i=1}^{n}\left|x_{i}\right|$. Let $X_{a}:=\left\{x \in \mathbb{R}^{n}\left|\exists x_{i}:\right| x_{i} \mid<a\right\}$. Then we have:

$$
\int_{\mathbb{R}^{n} \backslash X_{a}} f \circ P d x=2^{n} \int_{a^{n}}^{\infty} \frac{f(p)}{(n-1) !} \log \left(\frac{p}{a^{n}}\right)^{n-1} d p
$$


Proof: We prove this by induction. If $n=1$ the equality is easily verified. Now:

$$
\begin{aligned}
& \int_{\mathbb{R}^{n} \backslash X_{a}} f \circ P d x \\
& \left.=2^{n} \int_{a}^{\infty}\left(\int_{a}^{\infty} \cdots \int_{a}^{\infty} f\left(x_{1} \cdot \ldots \cdot x_{n}\right)\right) d x_{1} \cdots d x_{n-1}\right) d x_{n} \\
& =2^{n} \int_{a}^{\infty}\left(\int_{a^{n-1}}^{\infty} \frac{f\left(x_{n} \cdot q\right)}{(n-2) !} \log \left(\frac{q}{a^{n-1}}\right)^{n-2} d q\right) d x_{n} \\
& =2^{n} \int_{a^{n}}^{\infty} f(p)\left(\int_{a}^{\frac{p}{a^{n-1}}} \frac{1}{(n-2) !}\left[\log \left(\frac{p}{x_{n} a^{n-1}}\right)\right]^{n-2} \frac{1}{x_{n}} d x_{n}\right) d p \\
& =2^{n} \int_{a^{n}}^{\infty} f(p)\left(\frac{1}{(n-2) !}\left[\sum_{k=0}^{n-2}\left(\begin{array}{c}
n-2 \\
k
\end{array}\right) \log \left(\frac{p}{a^{n-1}}\right)^{k} \int_{a}^{\frac{p}{a^{n-1}}}\left(-\log \left(x_{n}\right)\right)^{n-k-2} \frac{1}{x_{n}} d x_{n}\right]\right) d p \\
& =2^{n} \int_{a^{n}}^{\infty} \frac{f(p)}{(n-1) !}\left(\left[-\sum_{k=0}^{n-2}\left(\begin{array}{c}
n-1 \\
k
\end{array}\right) \log \left(\frac{p}{a^{n-1}}\right)^{k}\left(-\log \left(\frac{p}{a^{n-1}}\right)\right)^{n-k-1}\right]\right. \\
& \left.+\left[\sum_{k=0}^{n-2}\left(\begin{array}{c}
n-1 \\
k
\end{array}\right) \log \left(\frac{p}{a^{n-1}}\right)^{k}(-\log (a))^{n-k-1}\right]\right) d p \\
& =2^{n} \int_{a^{n}}^{\infty} \frac{f(p)}{(n-1) !}\left(-\left[\log \left(\frac{p}{a^{n-1}}\right)-\log \left(\frac{p}{a^{n-1}}\right)^{n-1}+\log \left(\frac{p}{a^{n-1}}\right)^{n-1}\right]\right. \\
& \left.+\left[\log \left(\frac{p}{a^{n-1}}\right)-\log (a)^{n-1}-\log \left(\frac{p}{a^{n-1}}\right)^{n-1}\right]\right) d p \\
& =2^{n} \int_{a^{n}}^{\infty} \frac{f(p)}{(n-1) !} \log \left(\frac{p}{a^{n}}\right)^{n-1} d p .
\end{aligned}
$$

We compute the lower bound as follows. Apply the Feynman-Kac formula for $Z_{Q}(t)$ to obtain:

$$
Z_{Q}(t)=(4 \pi t)^{-n / 2} \int_{x \in \mathbb{R}^{n}} \mathbb{E}_{x, x ; 2 t}\left[\exp \left(-\int_{0}^{2 t} \frac{1}{2} \prod_{i=1}^{n}\left|b_{i}(s)\right|^{\alpha_{0}} d s\right)\right] d x,
$$

Now, remove some of the points $x=\left(x_{1}, \ldots, x_{n}\right)$ in the integral, as well as some Brownian paths. Only keep those points $x$ that satisfy $x \notin X_{t^{1 / 2}(\ln t)^{2}}$. Only keep Brownian paths with $\sup _{0 \leq s \leq 2 t}\left|b_{i}(s)-x_{i}\right| \leq t^{1 / 2}|\ln (t)|, \forall 1 \leq i \leq n$, where we remind again that in our notation $b_{i}(0)=x_{i}$.

The measure of such paths is $1-\rho(t)$, where $\rho(t) \sim e^{-D\left(\ln (t)^{2}\right)}$ as $t \rightarrow 0^{+}$. Since $P(x)=\prod_{i=1}^{n}\left|x_{i}\right|$, we have on points $x \in \mathbb{R}^{n}$ with $x_{i} \neq 0, \forall i$ :

$$
\frac{\partial \ln P(x)}{\partial x_{i}}=\frac{1}{x_{i}}, \quad \forall i
$$


Therefore, everywhere along the remaining paths we get by Taylor's formula:

$$
\begin{aligned}
|\ln P(b(t))-\ln P(x)| & =\left|D(\ln P)_{\mid x}(b(t)-x)+o(|| b(t)-x||)\right| \\
& \leq c /|\ln (t)| .
\end{aligned}
$$

Thus, with $\kappa(t):=\exp (c / \ln (t))$ we have: $P(b(t)) \leq \kappa(t) P(x)$. Inserting this into the expectation value, the integrand in the $s$-integral is no longer dependant on the Brownian motion. Thus:

$$
\begin{aligned}
\operatorname{Tr}\left(e^{-t H_{n}^{\alpha}}\right) & \geq \frac{1-\rho(t)}{(4 \pi t)^{n / 2}} \int_{\mathbb{R}^{n} \backslash X_{t^{1 / 2} \ln (t)^{2}}} e^{-t \kappa^{\alpha_{0}} P\left(x_{1}, \ldots, x_{n}\right)^{\alpha_{0}}} d x_{1} \cdots d x_{n} \\
& =\frac{1-\rho(t)}{(\pi t)^{n / 2}} \int_{t^{n / 2} \ln (t)^{2 n}}^{\infty} \frac{e^{-t \kappa^{\alpha} p^{\alpha} 0}}{(n-1) !} \ln \left(\frac{p}{t^{n / 2} \ln (t)^{2 n}}\right)^{n-1} d p \\
& =\frac{1-\rho(t)}{\kappa t^{\alpha_{0}^{-1}}(\pi t)^{n / 2}} \int_{t^{n / 2+\alpha_{0}^{-1}} \ln (t)^{2 n} \kappa}^{\infty} \frac{e^{-w^{\alpha} 0}}{(n-1) !} \ln \left(\frac{w}{\kappa t^{n / 2+\alpha_{0}^{-1}} \ln (t)^{2 n}}\right)^{n-1} d w
\end{aligned}
$$

Since $\rho \rightarrow 0$ and $\kappa \rightarrow 1$ as $t \rightarrow 0^{+}$we obtain:

$\liminf _{t \rightarrow 0} t^{n / 2+\alpha_{0}^{-1}}\left(\ln \left(t^{-n / 2-\alpha_{0}^{-1}}\right)\right)^{-(n-1)} \operatorname{Tr}\left(e^{-t H_{n}^{\alpha}}\right) \geq \frac{1}{\pi^{n / 2}(n-1) !} \int_{0}^{\infty} e^{-w^{\alpha_{0}}} d w$.

The Integral is equal to $\left(\alpha_{0}^{-1}\right) \Gamma\left(\alpha_{0}^{-1}\right)=\Gamma\left(1+\alpha_{0}^{-1}\right)$ and since $\ln \left(t^{-n / 2-\alpha_{0}^{-1}}\right)=$ $\left(n / 2+\alpha_{0}^{-1}\right) \ln \left(t^{-1}\right)$, we now conclude:

$$
\liminf _{t \rightarrow 0} t^{n / 2+\alpha_{0}^{-1}}\left(\ln \left(t^{-1}\right)\right)^{-(n-1)} \operatorname{Tr}\left(e^{-t H_{n}^{\alpha}}\right) \geq \frac{\Gamma\left(1+\alpha_{0}^{-1}\right)\left(n / 2+\alpha_{0}^{-1}\right)^{(n-1)}}{\pi^{n / 2}(n-1) !} .
$$

So lower and upper bound agree to first order and the theorem is proven.

Acknowledgments. The authors thank Werner Kirsch and Brice Franke for explaining us Feynman-Kac representations of heat-kernels/operators and the Ito-calculus. Both authors were supported by the project SFB-TR12, Symmetries and Universality in Mesoscopic Systems founded by the DFG.

\section{References}

[1] J. Aramaki and A. Nurmuhammad, A note on non-classical eigenvalue asymptotics. Hokkaido Mathematical Journal Vol.30 (2001) p. 307-325

[2] F.A. Berezin and M.A. Shubin, The Schrödinger equation. Mathematics and its Applications (Soviet Series) (1991). 
[3] M.S. Birman and M.Z. Solomyak, The principal term of spectral asymptotics for non-smooth elliptic problems. Funktsional. Anal. i Prilozhen. 4:4 (1970), 113 (Russian), English transl. in Functional Anal. Appl. 4 (1971).

[4] R. Brummelhuis, T. Paul and A. Uribe, Spectral estimates around a critical level. Duke Math. J. 78 (1995) no.3, 477-530.

[5] B. Camus, Equilibrium and eigenfunctions estimates in the semiclassical regime. J. Math. Phys. 47 (2006), no. 11.

[6] Y. Colin de Verdière, Ergodicité et fonctions propres du laplacien. Comm. Math. Phys. 102 (1985), 497-502.

[7] P. Exner and D. Barseghyan, Spectral estimates for a class of Schrödinger operators with infinite volume phase space and potential unbounded from below. J. Phys. A: Math. Theor. 45 (7) (2012)

[8] M. Kac, Can One Hear the Shape of a Drum? American Mathematical Monthly 73 (1966) (4, part 2).

[9] D. Robert, Comportement asymptotique des valeurs propres d'opérateurs du type Schrödinger à potentiel "dégénéré". J. Math Pures Appl. 61 (1982), 275-300.

[10] B. Simon, Functional integration and quantum physics. Pure and Applied Mathematics 86, Academic Press Inc., New York (1979).

[11] B. Simon, Some quantum operators with discrete spectrum but classically continuous spectrum. Ann. Physics 146 (1983), no. 1, 209-220.

[12] B. Simon, Nonclassical eigenvalue asymptotics. J. Funct. Anal. 53 (1983), no. $1,84-98$.

[13] M.Z. Solomyak, Asymptotic behavior of the spectrum of the Schrödinger operator with nonregular homogeneous potential. Mat. Sb. (N.S.) 127(169) (1985), no. 1, 21-39.

[14] H. Weyl, Über die asymptotische Verteilung der Eigenwerte. Nachrichten der Königlichen Gesellschaft der Wissenschaften zu Göttingen, (1911), 110117. 